\title{
Polyfunctional Nanofibril Appendages Mediate Attachment, Filamentation, and Filament Adaptability in Leptothrix cholodnii
}

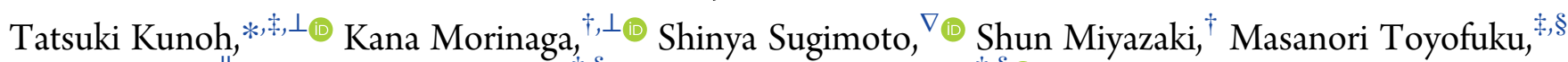
Kenji Iwasaki," Nobuhiko Nomura, ${ }^{*},+$, and Andrew S. Utada $*,+, \S(0)$

${ }^{\ddagger}$ Faculty and ${ }^{\dagger}$ Graduate School of Life and Environmental Sciences, ${ }^{\S}$ Microbiology Research Center for Sustainability, and "Life Science Center for Survival Dynamics, Tsukuba Advanced Research Alliance, University of Tsukuba, 1-1-1 Tennodai, Tsukuba, Ibaraki 305-8577, Japan

${ }^{\nabla}$ Department of Bacteriology and Jikei Center for Biofilm Research and Technology, The Jikei University School of Medicine, 3-25-8, Nishi-Shimbashi, Minato-ku, Tokyo 105-8461, Japan

Supporting Information

ABSTRACT: Leptothrix is a species of Fe/Mn-oxidizing bacteria known to form long filaments composed of chains of cells that eventually produce a rigid tube surrounding the filament. Prior to the formation of this brittle microtube, Leptothrix cells secrete hair-like structures from the cell surface, called nanofibrils, which develop into a soft sheath that surrounds the filament. To clarify the

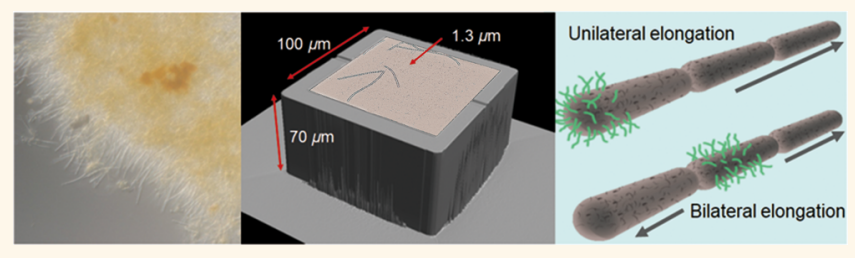
role of nanofibrils in filament formation in L. cholodnii SP-6, we analyze the behavior of individual cells and multicellular filaments in high-aspect ratio microfluidic chambers using time-lapse and intermittent in situ fluorescent staining of nanofibrils, complemented with atmospheric scanning electron microscopy. We show that in SP-6 nanofibrils are important for attachment and their distribution on young filaments post-attachment is correlated to the directionality of filament elongation. Elongating filaments demonstrate a surprising ability to adapt to their physical environment by changing direction when they encounter obstacles: they bend or reverse direction depending on the angle of the collision. We show that the forces involved in the collision can be used to predict the behavior of filament. Finally, we show that as filaments grow in length, the older region becomes confined by the sheath, while the newly secreted nanofibrils at the leading edge of the filament form a loose, divergent, structure from which cells periodically escape.

KEYWORDS: filamentous bacterium, Leptothrix, two-dimensional microfluidics, nanofibrils, adhesion

$\mathrm{M}$ icrobial mats are macroscopic biofilms made of stratified communities of microorganisms that develop in mineral enriched aqueous environments, where they typically grow at the solid-liquid interface of stream beds. Like biofilms, these communities facilitate the sharing of resources, provide protection from predation ${ }^{1,2}$ and changing environmental conditions, and enable synchronization of gene expression for their inhabitants. ${ }^{3-5}$ Whereas most biofilms are densely packed, microbial mats are fundamentally different; these communities are porous structures composed of flocs woven together with extracellular polymeric substances (EPS) and rigid bacterial stalks and sheaths. ${ }^{2,5,6}$ Microbial mats have received much less attention than traditional surface attached biofilm communities despite their significant role in the global cycling of minerals. ${ }^{6}$

The sheath-forming genus Leptothrix is a diverse group of bacteria that frequently dominate microbial mats in iron-rich aqueous environments. ${ }^{7}$ These bacteria form linear cell-chains surrounded by an EPS sheath that can reach $\sim 100$ s of micrometers in length. ${ }^{2,8}$ In the process of filament formation, the bacteria secrete proteins that oxidize dissolved $\mathrm{Fe}$ and $\mathrm{Mn}$, which in turn generates metal oxide nanoparticles that accumulate in the sheath matrix; ${ }^{7,9-11}$ this process transforms the soft sheath into a rigid mineral-encrusted hollow microtube, $\sim 1.5 \mu \mathrm{m}$ in diameter. These rigid microtubes play important physical as well as ecological roles: they provide mechanical support for mat development, while simultaneously facilitating intramat migration of inhabitants toward more favorable environments. ${ }^{2,8,10}$ In addition to structural support, the linear organization of these filamentous bacteria may indicate longrange electron or signal transport. For example, remarkable filamentous bacteria from the genus Desulfobulbaceae, known as "cable bacteria", transmit electrons intercellularly over centimeters within an insulating sheath. ${ }^{12-14}$ Whether Leptothrix

Received: June 13, 2019

Accepted: November 20, 2019

Published: December 5, 2019 
possesses a similar transport mechanism within their sheath is unknown.

The sheaths themselves contain abundant $\mathrm{COOH}, \mathrm{NH}_{2}$, and $\mathrm{SH}$ terminal functional groups that can act as a passive matrix that sorbs metals. ${ }^{10,15-20}$ These reactive end-groups have been implicated in the biomineralization of a broad range of commercially important metals such as $\mathrm{Ag}, \mathrm{Au}, \mathrm{Cu}, \mathrm{Rh}, \mathrm{Ru}$, $\mathrm{Ti}, \mathrm{Y}$, and $\mathrm{Zr}^{20,21} \mathrm{~A}$ fundamental understanding of sheath formation on filaments could enable the industrial harvesting of pigments and catalysts, lead to the development of novel amorphous iron oxides for lithium-ion battery anodes, and facilitate extraction of heavy and precious metals from surface waters through development of novel absorbents from the sheaths. ${ }^{11,20-24}$

The type species of the Leptothrix genus, L. ochracea, is a prolific sheath forming bacterium. However, it has proven resistant to laboratory cultivation due to intricate ecological interdependencies, ${ }^{25}$ making real-time analysis of sheath development difficult. ${ }^{8,26}$ Although there appear to be differences in the respective mechanisms of sheath formation, the related bacterium $L$. cholodnii has been used as a model organism to clarify much of what is known about sheath formation due to the ease of its cultivation. ${ }^{1,27}$ Briefly, the $L$. cholodnii sheath gradually develops from physical entanglements, or possibly chemical cross-links, ${ }^{1,28}$ between hair-like appendages that decorate the catenulate cells. These appendages, called nanofibrils, are glycoconjugates, which are mainly composed of complex amino-polysaccharides ${ }^{19}$ that are $\sim 10 \mathrm{~nm}$ in diameter and $\sim 10 \mu \mathrm{m}$ in length. ${ }^{8,24,27}$ As cell division proceeds, new nanofibrils are continuously secreted and the entanglements are thought to irreversibly aggregate, eventually enclosing the cell filament in a sheath of woven nanofibrils. ${ }^{1,24,27}$ Previous studies have clarified much of the qualitative description of sheath formation using a combination of traditional live-cell imaging methods in Petri dish cultures, ${ }^{1,28,29}$ while atomic force microscopy (AFM), ${ }^{8}$ scanning electron microscopy (SEM), ${ }^{30}$ and transmission electron microscopy (TEM) ${ }^{7,27}$ have been used to characterize the nanostructure of the sheath and microtubes. However, the role of nanofibrils in surface recognition, filament initiation, and sheath development remains unclear. Despite the importance of these different imaging methods, which have led to this qualitative picture, they are unable to provide access to the spatial and temporal resolutions required to analyze the behavior of the individual filament-founding bacteria. ${ }^{31-33}$ This is crucial to elucidating the mechanisms of sheath and filament formation in these bacteria.

In this paper, we investigate the function of $L$. cholodnii nanofibrils in the context of surface attachment and filament formation using time-lapse microscopy in high-aspect ratio twodimensional (2D) microfluidic culture chambers complemented with atmospheric scanning electron microscopy (ASEM)..$^{34-36}$ Our microfluidic device allows us to circumvent the difficulties in tracking development of a bacterial colony that rapidly forms massive three-dimensional (3D) structures by directing growth in 2D, while simultaneously enabling multiple labeling and washing steps without disturbing the sample. We show that cellassociated nanofibrils are important for surface attachment and that their distribution on young filaments is correlated to the direction of filament elongation. We show that elongating filaments demonstrate the ability to adapt to obstructions by bending in a new direction or by reversing the direction of elongation; this behavior depends on the balance of bending and compressive forces experienced by the tip of the filament due to the angle of impact. Using ASEM we show that the nanofibril sheath envelops cell filaments as early as the second division, which limits cell escape to the elongating distal pole. Furthermore, we show that the nanofibril sheath displays a density gradient as a function of axial position, corresponding to the degree of sheath maturation. Near the initial attachment point, where the sheath is most mature, it has a dense tubular structure, whereas close to the distal tip, the relatively immature sheath has a diffuse, divergent structure. When cell growth outpaces sheath maturation, this divergent structure may be unable to retain the cells, resulting in cell-escape from the open end of the sheath.

\section{RESULTS AND DISCUSSION}

SP-6 Surface Attachment and Filament Formation in 2D Chambers. Leptothrix is known to form amorphous multicellular aggregates ranging in extent from centimeters to meters in the environment as well as in batch culture. ${ }^{7,16,24,37} \mathrm{~L}$. cholodnii SP-6 cells grown in 2 day batch-culture, similarly, develop into such 3D aggregates (see SI Figure S1). Confocal images of representative aggregates reveal that they are mainly composed of heavily entangled filaments. Due to the difficulty in analyzing dense 3D structures, which simultaneously obscure the dynamics of individual cells and the developmental progression of individual filaments, we utilize a microfluidic device to culture individual cells in high aspect-ratio "2D" chambers. Since the height of the chambers is only slightly larger than the diameter of SP-6 cells, the cells and filaments are always in focus and adjacent filaments are unable to grow over one another; this enables the tracking of filament evolution (see TOC graphic and schematic shown in SI Figure S2). A video of cells growing in a "2D" chamber is shown in SI Movie S1. In contrast, a video of several cells at the bottom of a glass-bottom dish demonstrates the difficulty in imaging unconfined cells; here some cells diffuse randomly, another demonstrates tethered swimming, ${ }^{38,39}$ while another has attached to the surface (SI Movie S2).

We inoculate the device by infusing bacterial culture into the microfluidic channels, which typically carries a few cells into the chambers. Once there, cells swim about the chamber using a polar flagellum ${ }^{7,40}$ before irreversibly ${ }^{41-43}$ attaching to the surface, which we define as the starting time of the experiment $(t$ $=0 \mathrm{~min}$ ). Unattached cells are washed out of the device due to the flowing medium. We observe two distinct modes of elongation in the chambers: (1) unilateral, where a single pole of the filament elongates due to cell division, while the other pole remains fixed in the chamber; and (2) bilateral, where both poles simultaneously elongate, while the center of the founder cell remains fixed in the chamber. SP-6 cells exhibiting unilateral and bilateral elongation are shown in the time-lapse image sequences in Figure 1A,B, respectively. A kymograph showing the smooth elongation of a single filament is shown in SI Figure S3. In contrast, the "sheathless" variant SP-6 strain (SP-6 SL), which is unable to biosynthesize the sheath matrix, ${ }^{7}$ lacks the ability to attach within the $2 \mathrm{D}$ chambers for the duration of the experiments. Although SL variants are reported to be able to form filaments in static conditions, ${ }^{7}$ we find that filament formation is ablated; these cells simply roam about the chamber, periodically dividing, as shown in SI Movie S3 and Figure 1C. Aside from cellular elongation due to division, the average length of the SL cells remains constant at $\sim 4 \mu \mathrm{m}$ over the course of the experiment. For the same time period, surface-attached SP-6 

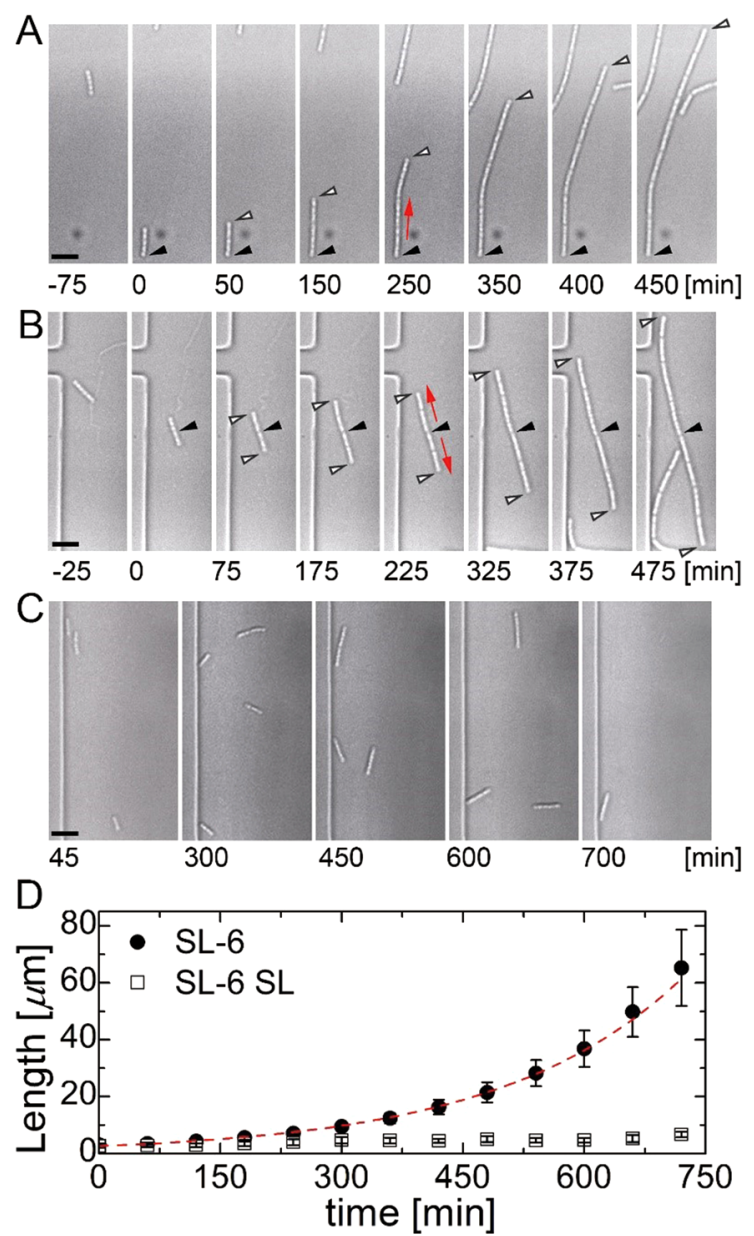

Figure 1. Time-lapse sequence showing cell growth in 2D chambers for cells exhibiting (A) unilateral elongation and (B) bilateral elongation. (C) Time-lapse sequence of a variant strain (SP-6 SL) unable to produce a sheath. Black arrowheads in $(A, B)$ indicate the same spatial position in each image, the unfilled arrowheads indicate the position of the moving pole(s), and red arrows indicate the direction of elongation. Scale bars $=5 \mu \mathrm{m}$. The numbers refer to the time in minutes. (D) Filament length of SP-6 (๑) and SP-6 SL ( $\square$ ) strains measured as a function of time within 2D chambers. The error bars represent the standard deviation (SD) taken from 10 filaments. The dashed exponential line is a best fit to the data (see Supporting Material and Methods S1.3 section).

cells form cell filaments that increase in length monotonically. Assuming exponential cell growth within the filament, we fit the length with an exponential and find good agreement over this limited time frame (Figure 1D).

These results indicate that cell-surface attachment is necessary for filament formation. They also suggest that, based on the differences between SP-6 and SL variants, the ability to synthesize a sheath appears to play a major role in surface attachment. This is not surprising since the sheaths of other species of Leptothrix are known to be adherent to surfaces, cells, and other filaments. ${ }^{8,10,37}$ However, since surface attachment and time appear to be important for sheath development, it is likely that the surface attaching SP-6 cells are decorated with an incompletely formed sheath, composed of entangled nanofibrils; this suggests a direct role for nanofibrils in surface attachment. Importantly, we note that although SL variants do not synthesize a sheath, it is unknown if they can generate nanofibrils. In fact, the sheathless variant of a different $L$. cholodnii (OUMS1) strain can produce nanofibrils; however, in this case, the nanofibrils disassociate from the cells. ${ }^{18,30}$ Thus, it is unknown if SP-6 SL variants do not produce a sufficient quantity of nanofibrils or if they are somehow lost. Taken together, these data suggest that in the case of SP-6, cell surface-associated nanofibrils appear to be important for initial surface attachment.

Direction of Elongation and the Distribution of Nanofibrils on Young Filaments. To clarify the role of the cell-associated nanofibrils in the unilateral and bilateral modes of elongation, we fluorescently label the nanofibril aggregates with an $\mathrm{NH}_{2}$-specific fluorescein reagent on live cells in batch culture $^{19}$ (see SI Figure S4A). We confirm the specificity of the fluorescein label by co-staining with a lectin, which recognizes sugar molecules contained in the nanofibrils. The overlap of fluorescence signals strongly suggests that the $\mathrm{NH}_{2}$-specific reagent is sufficiently specific to the nanofibrils (see Materials and Methods and SI Figure S5).

We find that SP-6 aggregates strongly fluoresce when labeled with the fluorescein reagent, indicating an abundance of nanofibrils, whereas SP-6 SL cells exposed to the same conditions remain unlabeled (see SI Figures S4B and S5). Filtering the batch culture to separate the large SP-6 aggregates from planktonic cells, we find that the free individuals in the filtrate have little to no fluorescence compared to the aggregates from the same culture. This difference in fluorescence suggests that prior to surface attachment, the free SP-6 cells in batch culture lack a sufficient amount of nanofibrils to be labeled; this result parallels the lack of fluorescence in the SP-6 SL cells (see SI Figure S4B, second and third columns).

To track the secretion of nanofibrils on these young SP-6 filaments from the filtrate, we culture cells within the " $2 \mathrm{D}$ " microfluidic chambers, then label their nanofibrils. During this process, we incubate the cells long enough for them to lengthen by at least $\sim 50 \%$ prior to infusing the labeling reagent to allow sufficient accumulation of the nanofibrils to be labeled (see Materials and Methods). We find that unilaterally elongating filaments acquire fluorescence around the stationary pole, while the elongating pole remains unlabeled. In contrast, bilaterally elongating filaments acquire fluorescence in a central band, while the elongating poles remain unlabeled; this central band is centered around the location of the mother cell. An image sequence of uni- and bilaterally elongating filaments combined with fluorescence labeling is shown in Figure 2A. In contrast, cells that arrive in the " $2 \mathrm{D}$ " chamber immediately prior to staining, conspicuously, remain free of fluorescence (see circled cells in Figure 2A, $t=200 \mathrm{~min}$ ). Based on the width of the fluorescence on the labeled cells, it appears that $>100 \mathrm{~min}$ are needed for the biosynthesis of a sufficient quantity of the nanofibrils to be labeled (see Figure $2 \mathrm{~A}, t \geq 215 \mathrm{~min}$ ). Although we lack the spatial resolution to determine the $3 \mathrm{D}$ distribution of nanofibrils on individual cells, the fluorescence distribution on young filaments suggests that during unilateral elongation, the nanofibrils close one end of the sheath, whereas during bilateral elongation, nanofibrils are more centrally located, leaving both ends free to elongate.

Fluoresence labeling of the entangled nanofibrils enables the visualization of their approximate distribution on young filaments but is unable to access the submicron distribution on individual cells. To do so, we turn to ASEM, which can be used to image directly in aqueous solutions and thus is free from the artifacts associated with sample dehydration. ${ }^{24}$ ASEM reveals that SP-6 cells are heterogeneously covered with nanofibrils, while in stark contrast, the sheathless variants lack visible 


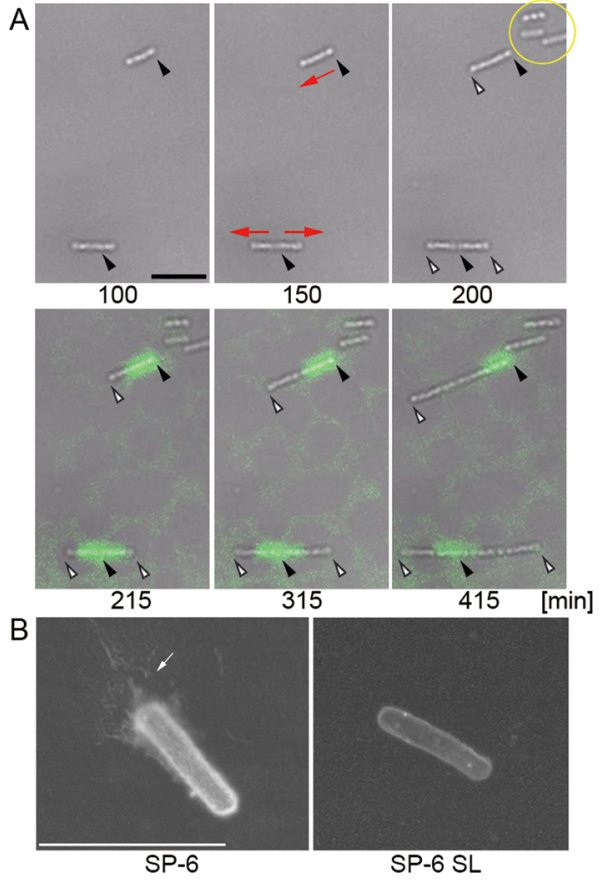

Figure 2. Cellular distribution of nanofibrils. (A) Bright-field and fluorescence time-lapse sequence with stop-flow fluorescent labeling of the nanofibrils during that gap between 200 and $215 \mathrm{~min}$. The upper cell demonstrates unilateral elongation, while the lower cell demonstrates bilateral elongation. Black arrowheads indicate the same spatial position in each image, red arrows indicate the direction of elongation, the yellow circle encloses cells that attach in the chamber midway through the imaging sequence, and the unfilled arrowheads indicate the position(s) of the moving pole(s). The numbers refer to the time in minutes. (B) ASEM images of SP-6 (left) and SP-6 SL (right) cells. The white arrow (left) indicates the nanofibrils. Scale bars $=5 \mu \mathrm{m}$.

nanofibrils altogether; representative ASEM images of SP-6 and SP-6 SL cells acquired under the same settings are shown in Figure 2B and SI Figure S6. The absence of fluorescently labeled nanofibrils on free SP-6 cells (SI Figure S4B) and the lack of nanofibrils on SL cells in the ASEM images (Figure 2B) collectively indicate that cell-associated nanofibrils are important for surface attachment.

Collisions between Filaments and Obstructions Lead to Bending and Reversal. In the absence of obstacles, elongating filaments grow approximately linearly. However, due to the finite dimensions of the chamber, growing filaments frequently collide with obstacles such as the chamber wall or other filaments. Obstacles can either deflect the direction of elongation, which causes filaments to bend, or they directly impede forward progress, which causes the direction of elongation to reverse, as shown in Figure 3A,B, respectively (see also SI Movies S4 and S5). Tracking filament evolution as a function of time reveals that the precollision shape in both bending and reversing filaments is unaffected by an impact (see colored traces overlaid in Figure 3A,B). In addition, we observe that elongating filament poles are unable to dislodge established sections of other filaments (see SI Movie S1). Axially compressed rods will generally undergo long-wavelength buckling unless the shaft is supported, when it can support higher curvature bends. ${ }^{44}$ Since collisions appear to have no effect on the precollision shape, it appears that the filaments are supported against lateral deflection; this could be effected through attachment of the external face of the sheath to the chamber, while the cells move through the inner tube.

To elucidate the causes of filament bending versus reversal, we consider the component forces experienced by a filament during a collision. Filament elongation could arise from a number of sources such as an uncharacterized surface motility or through nanofibril extrusion that propels the enclosed cells forward similar to a model proposed for L. ochracea. ${ }^{8}$ Although we do not rule out these other forces, it appears that SP-6 filament elongation is largely driven by cell division, directed away from the initial patch of attachment. The location of initial attachment likely harbors the oldest secreted nanofibrils and thus likely has the strongest adhesion energy. ${ }^{45}$ We assume that during a collision, filament compression results from cellular division of the catenulate cells confined within the sheath, which pushes axially away from the initial attachment point. These assumptions limit the maximum force that a filament can exert using its adhesive patch of entangled nanofibrils at the initial attachment point, $F_{\text {adh }}$. During a collision, filaments experience varying degrees of bending and compressive loads depending on the angle of the impact. Forces directed perpendicularly to the axis of the filament act to bend the filament, while forces directed along the filament axis act to compress the filament, which can cause reversal. We define these forces as $f_{\text {bend }}=F_{\text {adh }} \sin \theta \cos \theta$ and $f_{\text {reverse }}=F_{\text {adh }}\left(\sin ^{2} \theta-\cos ^{2} \theta\right)$, respectively, where $\theta$ is the angle between the filament axis and the obstacle, for $0^{\circ} \leq \theta \leq$ $90^{\circ}$ (see inset in Figure 3C, SI S1.4, and SI Figure S7). We assume that filaments have an inherent stiffness that resists bending, ${ }^{8,46}$ while their nanofibril at the initial attachment point resists reversal during a collision with an obstacle; however, when the bending or compressive force on the filament surpasses these resistive forces, they will either bend or reverse, respectively.

To describe the state of the system, we normalize all forces by $F_{\text {adh }}$ and plot $f_{\text {bend }}$ and $f_{\text {reverse }}$ as a function of $\theta$, as shown in Figure 3C. According to this simple model, bending becomes possible as $f_{\text {bend }}$ becomes comparable to the bending stiffness for $\theta \gtrsim 10^{\circ}$, whereas filament reversal becomes possible as $f_{\text {reverse }}$ becomes comparable to $F_{\text {adh }}$ for $\theta \gtrsim 75^{\circ}$. In addition, in the range of $\theta \approx 65-80^{\circ}$, there are $\theta$ values where both bending and reversal can occur. Finally, as $\theta \rightarrow 90^{\circ}$, filament reversal is predicted to become dominant as $f_{\text {reverse }}$ approaches unity. We note that the relative values of filament stiffness and nanofibril adhesion force are adjustable in the model and determine the specific crossover $\theta$ values. Moreover, to account for population level heterogeneity in bending stiffness and nanofibril adhesion force, we incorporate variability into these resistive model forces.

To test this simple model, we quantify the actual outcome of collisions by measuring the frequencies of bending and reversal as a function of $\theta$. Supporting the general shape of the forces in the model, the probability densities show that bending occurs over a wide range of $\theta$ values, while filament reversals occur only when $\theta>60^{\circ}$ (Figure 3D). We simulate the outcomes of multiple collisions as a function of $\theta$ and generate probability densities for bending and reversal, shown by the dashed and dotted lines in Figure 3D (see SI S1.4). By adjusting the relative strength of the filament stiffness and nanofibril adhesive force as well as the variability of these resistive forces, we achieve good agreement between the data and the model. Surprisingly, when we remove all variability from the filament bending stiffness (blue filled area in Figure 3C), the model predicts the absence of filament bending for $\theta>75^{\circ}$, which contradicts our observations (line-filled bars, Figure 3D). In fact, the significant number of 

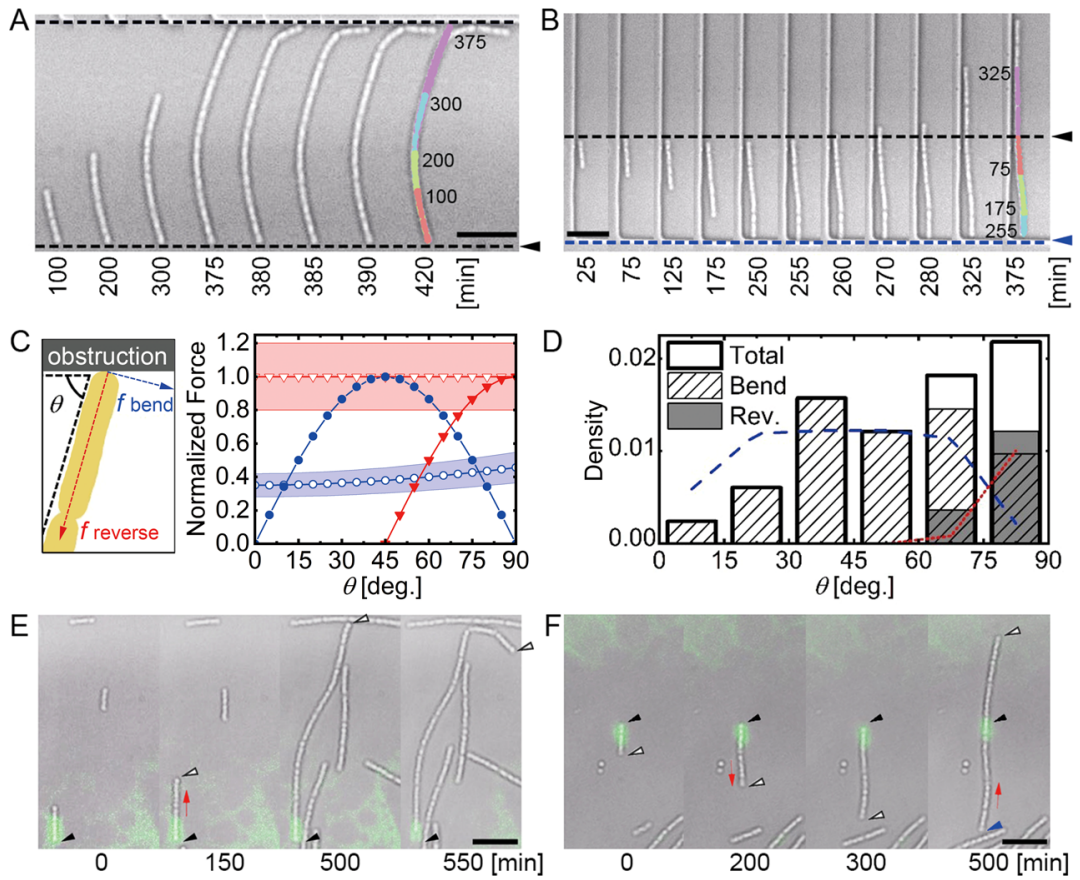

Figure 3. Filament bending and reversal. Time-lapse image sequence showing impact-induced filament (A) bending (at $t=380 \mathrm{~min})$ and (B) reversal (at $t=255 \mathrm{~min}$ ). The colored cells in (A,B) are overlays of the filament at different time points (labeled). (C) Model of the forces generated during a collision between a filament and obstacle as a function of the angle of impact, $\theta$. The blue circles $(\bullet)$ represent $f_{\text {bend }}$ the unfilled circles $(O)$ represent the bending stiffness, and the blue shaded area indicates $30 \%$ variability in this value. The red triangles $(\nabla)$ represent $f_{\text {reverse }}$, the unfilled triangles $(\nabla)$ represent the nanofibril adhesion force, $F_{\text {adh }}$, and the red shaded area indicates $20 \%$ variability. (Inset) Schematic showing the collision between an elongating filament and an obstruction. (D) Binned probability densities of bending and reversing as a function of $\boldsymbol{\theta}$. The sum of the respective densities for bending and reversal equal the total, shown by the thick black line outlining the bars. The dashed (blue) and dotted (red) lines are simulated probability densities for bending and reversal, respectively, based on the model in panel C (see SI S1.4). (E,F) A time-lapse sequence combined with stop-flow fluorescence labeling of nanofibrils showing impact-induced bending and reversal, respectively. For $(A, B) t=0$ defines the moment of cell-surface attachment, while in $(E, F)$ it refers to start of the experiment upon completion of the nanofibril labeling. In all time-lapse sequences, the black arrowheads indicate the same spatial position in each image, the red arrows indicate the direction of elongation, unfilled arrowheads indicate the position of the moving pole(s), and the blue arrowheads indicate the location of a collision that causes reversal. The numbers refer to the time in minutes. Scale bars $=5 \mu \mathrm{m}$.

filaments that bend for $\theta>75^{\circ}$ reflects heterogeneity in flexural rigidity of the filament. This heterogeneity could arise from the fact that the filament is composed of the sheath and enclosed cells, each of which have their own bending rigidities. According to recent reports, the flexural rigidity of the L. ochracea sheath ${ }^{8}$ is comparable to that of Escherichia coli and Bacillus subtilis cells. ${ }^{47,48}$ Assuming that the L. cholodnii sheath has a similar flexural rigidity, a large variability in bending rigidity of the filament could arise when bending occurs over the gap between two cells, where only the sheath supports the strain, compared to when both the sheath and cells are simultaneously bent.

As the data show, the probability of reversal increases when a larger fraction of the total collision force is converted into compressing the filament $\left(\theta>60^{\circ}\right)$. Since filaments do not buckle during collisions nor are the individual cells in the filament compressible, $f_{\text {reverse }}$ is exerted directly on the nanofibril attachments that link the filaments to the surface at the initial attachment point. We suspect that during reversals, the entangled nanofibril sheath is unable to support the compressive load and is either pulled from the cell body or from the surface of the device, or both. To examine the effect of an impact on the nanofibril distribution at the point of initial attachment, we label the nanofibrils immediately after irreversible attachment (as in Figure 2A) then wait for collisions to occur. We find that the location of the labeled nanofibrils on bending filaments remains largely unaltered by collisions since there is no retrograde motion of the filament (see Figure 3E). Careful examination of the labeled nanofibrils reveals a small measure of fluorescence intensity loss, which could be due to the reagent washing away or a mixing of the labeled nanofibrils into the surrounding sheath as it matures. During reversal, we find a small measure of vertical displacement of the labeled nanofibril sheath; however, relative to the freed filament that slides past the initial attachment point, the nanofibril sheath remains adhered to the chamber in largely the same position (see Figure $3 \mathrm{~F}$ ). This indicates that the connections between the body of the founder cell and the nanofibrils rupture during reversal.

These data support our picture that filament bending and reversal depends on the component forces of impact at the filament tip. Moreover, SP-6 filaments demonstrate the ability to support high-curvature bending without catastrophic fragmentation as well as sustain compressive loads that reverse the direction of elongation without buckling. These characteristics suggest that at the time of collision, the filaments are already wrapped within an elastic sheath. In the case of filament bending, the combination of sheath elasticity and intercellular gaps enables bending without breaking, while in the case of reversal, the sheath prevents the cell chain from buckling under a compressive pressure.

Gradient in Sheath Maturation Starting from Initial Attachment Point. It is unclear when the entangled nanofibrils aggregate to form the sheath that encloses the filaments; 
however, during filament elongation, we frequently observe that the intercellular space between adjacent cells develops into wide gaps. Although these gaps can be as large as $\sim 2.5 \mu \mathrm{m}$, which is roughly twice the cell diameter, the cells remain aligned, even during cell division (see Figure 4A). The transience of these gaps suggests the presence of a sheath that maintains alignment of the cells in the filaments.
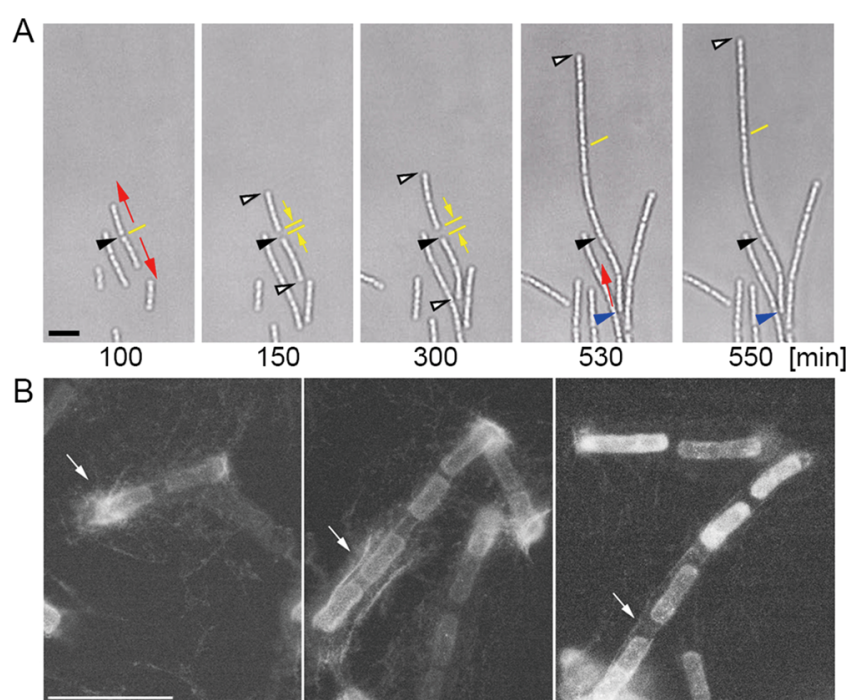

Figure 4. Intercellular gaps within filaments. (A) Time-lapse sequence showing the appearance and development of a large intercellular gap, followed by its disappearance during filament elongation. The black arrowheads indicate the same spatial position in each image, red arrows indicate direction of elongation, yellow lines and arrows indicate location and width of the gap between adjacent cells, the unfilled arrowheads indicate the position of the moving pole(s), and blue arrowheads indicate collision with an obstruction. The numbers refer to the time in minutes. (B) ASEM images showing the different stages of the immature sheath formation. White arrows indicate (left) diffuse nanofibrils on a young filament, (middle) a tighter distribution on a longer filament, and (right) the outline of the sheath on an even longer filament. Scale bars $=5 \mu \mathrm{m}$.

To directly image the state of the sheath, we again turn to ASEM. We culture SP-6 cells on an ASEM substrate long enough to ensure an abundance of filaments in various states of maturation. Although the young filaments, which are made of pairs of cells, tend to be surrounded by a diffuse web of nanofibrils, similar to the nanofibrils on individual cells, we find that the more mature filaments are surrounded by a tubular structure (compare Figure $2 \mathrm{~B}$ to $4 \mathrm{~B}$ ). Notably, pronounced intercellular gaps are also present in the longer filaments. These ASEM snapshots indicate that the nanofibril entanglements form a sheath as early as the second cellular division. Furthermore, taken together with the images from Figure 2, these results suggest a gradient in the degree of sheath maturation along the filament that corresponds directly to its shape: the oldest sections are tubular, while the younger sections are a diffuse web of nanofibrils.

Although the ASEM snapshots provide no information on the dynamics of sheath maturation, since we know that the sheath eventually develops into an open-ended biomineralized microtube, ${ }^{1,8}$ this suggests that the immature sheath itself is likely to be open-ended. Supporting this picture, cell escape occurs exclusively from the ends of the elongating filaments (Figure
$5 A)$. In light of the low nanofibril fluorescence intensity (Figure 2A, SI Figure S4) and the diffuse nanofibril distribution on both individual cells and young filaments (see Figures $2 \mathrm{~B}$ and $4 \mathrm{~B}$ ), we hypothesize that cells at the elongating poles are able to escape the filament due to insufficient nanofibril coverage and sheath development.

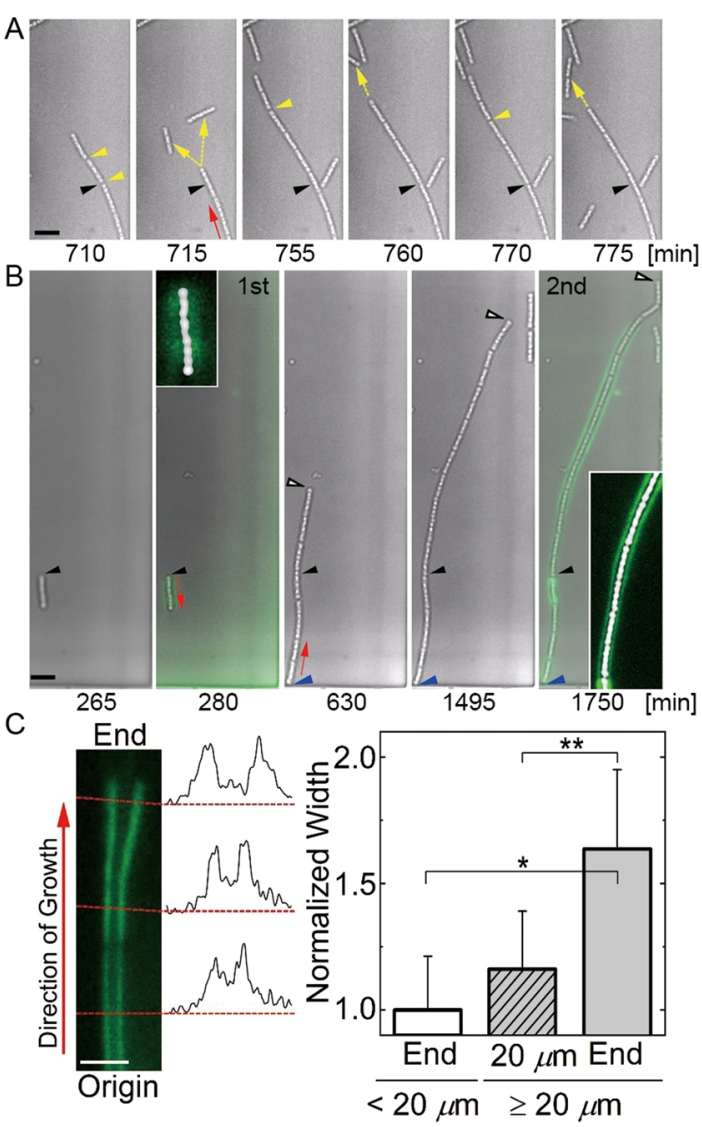

Figure 5. Filament fragmentation and development of the sheath. (A) Escape of cells from the leading edge of the filament. The yellow arrowheads indicate the positions where filament fragmentation occurs, whereas the yellow arrows indicate the positions of the cells, after escaping from the filament. (B) Time-lapse sequence with stop-flow fluorescence labeling of the nanofibrils at two time-points during 265-280 (first) and 1495-1510 min (second), respectively. (Insets) Magnified, background-subtracted images showing the distribution of fluorescent nanofibrils on the cell filament. For $(A, B)$ the black arrowheads indicate the same spatial position in each image, red arrows indicate the direction of elongation, blue arrowheads indicate the location of impact that causes reversal, unfilled arrowheads indicate the position of the moving pole $(\mathrm{s})$, and blue arrowheads indicate collision with an obstruction. (C) (left) Fluorescence intensity profile measured along the axis of a representative filament. The width is defined as the peak-to-peak distance between intensity maxima. (right) Histogram of intensityprofile widths measured at different axial positions normalized by the width of the filament at its origin. For filaments shorter than 20 $\mu \mathrm{m}$ in length, the width is measured at the distal end and normalized by the width at the origin (unfilled). For filaments $\geq 20 \mu \mathrm{m}$ in length, the widths are measured $20 \mu \mathrm{m}$ from the origin of filament growth (filled, striped) and at the distal end of the filament (filled), then normalized by the width measured at its origin. The error bars represent one SD measured from 6 to 9 filaments. The stars represent statistical significance: $(*)$ represents $p<0.01$ and $(* *)$ represents $p<0.02$. Scale bars $=5 \mu \mathrm{m}$. 
To test this hypothesis, we exploit the flow control afforded by the $2 \mathrm{D}$ microfluidic environment to visualize sheath evolution by applying two iterations of stop-flow nanofibril fluorescent labeling. The first staining labels the initial distribution, while the second staining labels subsequently secreted nanofibrils (see Figure $5 \mathrm{~B}, t=280$ and $1750 \mathrm{~min}$ ). Surprisingly, the lateral extent of fluorescence surrounding the filament broadens in the direction of elongation (see Figure $5 \mathrm{~B}, t=1750 \mathrm{~min}$ ). To quantify this broadening, we measure the intensity line-profile perpendicularly to the filament axis as a function of the distance from the filament origin, which is defined as the point of initial attachment (see Figure 5C, left). We find that filaments with lengths $<20 \mu \mathrm{m}$ have line-profile widths that are approximately constant, which indicates that the sheath is cylindrical. Filaments with lengths $\geq 20 \mu \mathrm{m}$ are measured at two locations: (1) $20 \mu \mathrm{m}$ from the origin and (2) at the distal end. The width at $20 \mu \mathrm{m}$ has a cylindrical profile, similar to the shorter filaments, while the line-profile width measured at the distal end of the filament shows statistically significant broadening. A histogram of normalized intensity line-profiles along different filaments is shown in Figure 5C (right).

Although we are unable to image the degree of nanofibril entanglement, these data confirm the presence of a gradient in the organization of the nanofibrils as a function of filament length. This appears to correspond to a gradient of sheath maturity and is consistent with previous work that has shown that sheath synthesis occurs primarily at the elongating pole(s). ${ }^{1}$ However, unlike prior work, because we confine the filaments to enable time-lapse imaging of filament elongation, we are able capture instances where the sheath has yet to evolve its characteristic cylindrical shape $e^{1,2,7,8,11}$ as it matures. We speculate that as the cell train is driven forward, nanofibrils caught in entanglements would begin to experience tension along the axis of the filament; this pulling could lead to axial alignment of nanofibrils and simultaneously cause the older sections to "tighten" as they constrict around the cells. Atomic force microscopy (AFM) images of L. ochracea sheaths have shown that the nanofibrils can become aligned along the filament axis. ${ }^{8}$ The nanofibrils secreted earlier in filament development become tubes that constrain and align the enclosed cells. These tubular sheaths are able to support bending and prevent filament buckling under compressive loading. However, the freshly secreted nanofibrils at the elongating pole of the filament spread outward, possibly with a conical shape, which may be less effective in preventing cell escape (Figure 5A). Our results suggest that the gradient in sheath development, leading to insufficient confinement at the elongating pole of the filament, may be a general phenomenon found in other filament producing bacteria that exhibit cell escape from the elongating pole. ${ }^{1,2,20,49-52}$

\section{CONCLUSIONS}

Leptothrix plays an important role in the global iron cycle through iron oxidation in chalybeate freshwater. Their tendency to form entangled 3D aggregates has hindered analysis of the dynamics of filament formation in microbial mats. An understanding the bacterial filament lifestyle is important for understanding the behavior of this and other filamentous bacteria that consist of ecologically important groups. Our approach makes use of microfluidics due to its demonstrated utility in enabling precise control and structuring of microenvironments while facilitating imaging. ${ }^{53}$ Leveraging these features, we designed a $2 \mathrm{D}$ chamber that facilitated trapping, culturing, and imaging multiple filaments over many hours and complemented these experiments with ASEM snapshots. We have shown that the L. cholodnii SP-6 nanofibrils are crucial for initial surface attachment and their distribution on young filaments appears to influence the direction of filament elongation. As the entangled nanofibrils develop into a sheath, it enables filaments to negotiate obstructions as it matures around the catenulate cells.

Although the role of the nanofibrils and sheath is becoming clear, it is still incomplete. Our data support the idea that at each stage of filamentation they impart adaptability to the environment. The nanofibrils appear to enable planktonic cells to remain in favorable environments with strong currents, while the sheathless variant lacks this ability. Upon surface attachment and filamentation, the developing nanofibril sheath protects the enclosed cells from predation and toxic biomineralization. Whole filaments, which are monoclonal colonies, due to their flexibility and reversibility have the ability to adapt to obstacles, and thereby elongate continuously; this contrasts with the relative immobility of surface-attached biofilm colonies encased in an EPS matrix. These features may enable Leptothrix to become one of the dominant microorganisms in microbial mats.

This developing picture has allowed us to ask more specific questions about Leptothrix filament formation, which will be interesting to test in future work. For example, are there conditions where filamentation is unfavorable, and if so, is it linked to environmental prosperity or adversity? In these instances, the catenulate cells require a means of escape, and as we have shown, SP-6 appears to possess it. We can imagine scenarios where nutrient-poor conditions could result in slowed nanofibril synthesis, leading to escape due to ineffective retention of cells at the distal pole, and nutrient-rich conditions could result in rapid division where cell growth may surpass sheath development, again, leading to escape. The features of nanofibril synthesis and sheath structure appear to define possible behaviors of the bacteria and require further investigation.

Our results provide a framework for understanding the role of appendages in L. cholodnii filament formation and, more generally, the behavior of other filamentous bacteria, which have shown promise in wastewater treatment, ${ }^{54,55}$ bioremediation, ${ }^{24,54,56}$ and biomineralization ${ }^{20,57}$ applications. A deeper understanding of sheath formation combined with micropatterning to guide filament growth could enable the synthesis of individual, high-aspect ratio microtubes that may find application in the fabrication of microwires, biohybrid magnetic robots for targeted delivery, ${ }^{58-60}$ or as a novel bioseparation material. ${ }^{61}$

\section{MATERIALS AND METHODS}

Strains and Culturing Conditions. In this study, we used $L$. cholodnii strain SP-6 (ATCC 51168) or its derivative strain SP-6SL (ATCC 51169), which is unable to synthesize the sheath skeleton. ${ }^{7}$ We cultured these strains by transferring freeze stock to a MSVP agar plate, ${ }^{7,16}$ followed by incubation at room temperature (RT). After culturing for a week, we transferred $1-3$ single colonies to $25 \mathrm{~mL}$ of liquid MSVP medium and incubated in a reciprocating shaker at RT and $65 \mathrm{rpm}$ for an additional 2 days. Following liquid culturing, cells were used immediately.

Microfluidic Device Fabrication and In Situ Culturing. We prepared microfluidic channels in polydimethylsiloxane (PDMS) replicated from a KMPR 1035 (Microchem, Westborough, MA, USA) master fabricated with two different heights. This was achieved by performing standard lithography two times ${ }^{33,62,63}$ (see also SI S1.1). 
Briefly, we spin-coated a layer of photoresist and exposed through a photomask using a mask aligner (SÜSS MicroTec, Garching, Germany). Without developing the first layer, a second layer of photoresist was then deposited, and a second photomask was aligned to the first photoresist layer. We then exposed a second time and developed the two-level structure. The main channel dimensions are $500 \mu \mathrm{m}$ wide $\times 70 \mu \mathrm{m}$ deep, with a length of $\sim 5 \mathrm{~mm}$, and contain a linear array of rectangular pillars, aligned along the central axis of this channel. The pillar dimensions are $100 \mu \mathrm{m}$ wide $\times 70 \mu \mathrm{m}$ tall. At the base of each pillar, there are $5 \mu \mathrm{m}$ wide inlets and outlets; these entrances lead to a rectangular cavity with dimensions: $100 \mu \mathrm{m} \times 100$ $\mu \mathrm{m} \times \sim 1.3 \mu \mathrm{m}$ (see TOC graphic and schematic shown in SI Figure S2). After $\mathrm{O}_{2}$ plasma bonding the PDMS slab to glass, this cavity forms the quasi-2D chamber.

MSVP medium was infused into the PDMS devices using positive displacement syringe pumps (Harvard Apparatus, Holliston, MA, USA) at a rate of $100 \mu \mathrm{L} / \mathrm{h}$. We inoculated a sufficient amount of 2 day culture into the main channel to achieve a seeding density of approximately 510 cells per $2 \mathrm{D}$ chamber. Images were taken on an Axio Observer.Z1 (Carl Zeiss, Oberkochen, Germany) at 5 min intervals. The microscope stage was equipped with a heating unit capable of maintaining a stable temperature of $25 \pm 0.5{ }^{\circ} \mathrm{C}$ (TP-SQH26-C16N, TOKAI HIT, Fujinomiya, Japan).

Fluorescent Staining of Cytoplasmic Membrane and Nanofibril Amino Groups $\left(\mathrm{NH}_{2}\right)$. To confirm normal cell growth can occur within the $2 \mathrm{D}$ chambers, we labeled the plasma membrane of SP- 6 cells with FM4-64 (Thermo Fisher, Waltham, MA, USA) and monitored the behavior of trapped cells for $3 \mathrm{~h}$. We dissolved FM4-64 at a concentration of $0.5 \mu \mathrm{g} / \mathrm{mL}$ in MSVP medium and infused at a rate of $100 \mu \mathrm{L} / \mathrm{h}$. A time-lapse sequence of the stained cells is shown in SI Figure S8A. As filaments elongate due to cellular division, distinct lines become visible along the filament. Staining the cytoplasmic membrane shows that these marks correspond to the septa between adjacent cells (see SI Figure S8B).

To visualize the presence of immature sheath skeletons, we labeled the terminal $\mathrm{NH}_{2}$ groups found within the nanofibrils ${ }^{19}$ with fluorescein (Fluorescein Labeling Kit- $\mathrm{NH}_{2}$, DOJINDO, Kumamoto, Japan) (see SI Figure S4A). We stained SP-6 and SP-6 SL cells in 2 day liquid culture with the fluorescein reagent diluted 1:50 in medium, then incubated at $37^{\circ} \mathrm{C}$ for 15 min. ${ }^{18}$ The cells were collected, washed in MSVP medium, and then imaged using an Axio Observer.Z1 or LSM780 confocal laser scanning microscope (Carl Zeiss). Individual SP-6 cells were isolated from large cellular aggregates for fluorescence labeling by first filtering the 2 day culture with a $5 \mu \mathrm{m}$ pore syringe filter then using the same staining protocol. However, this method suffers from the drawback in that it will label all $\mathrm{NH}_{2}$-rich regions.

To corroborate the specificity of the fluorescein reagent, we used the lectin Concanavalin A conjugated to Alexa594 (Con A-Alexa) (Thermo Fisher) to label glycosaminoglycans ${ }^{64,65}$ found in the L. cholodnii nanofibril sheaths. The nanofibrils are made of structural glycoconjugates, which are a type of glycosaminoglycan. ${ }^{19,66}$ By co-staining first with fluorescein and then with Con A-Alexa, we measured the amount of fluorescence overlap between $\mathrm{NH}_{2}$-rich regions and sugars (SI Figure S5). The working concentration of Con A-Alexa was $50 \mu \mathrm{g} / \mathrm{mL}$. Con A appears to affect filament elongation in live-imaging experiments, so we used it simply to confirm label specificity.

To follow the development of the sheath skeletons that surround the cell filaments, we first inoculated cells into the $2 \mathrm{D}$ chambers, added 500 $\mu \mathrm{L}$ of MSVP containing the fluorescein reagent, at a 1:60 dilution, and then stopped the flow. We incubated the reagent mixture with the adherent cells in $2 \mathrm{D}$ chambers at RT for $15 \mathrm{~min}$. We then restarted the flow of medium and resumed further time-lapse imaging. Bright-field images were taken at $5 \mathrm{~min}$ intervals and fluorescent images were taken at $25 \mathrm{~min}$ intervals.

Atmospheric Scanning Electron Microscopy (ASEM). We used ASEM (JASM-6200, JEOL, Tokyo, Japan) to directly visualize cellassociated nanofibrils. ${ }^{67}$ We were able to directly image the nanofibrils and their localization without problems associated with dehydration of the sample ${ }^{24}$ since imaging can occur directly in an aqueous medium. We prepared the cells by transferring $3 \mathrm{~mL}$ of 2 day-culture to an ASEM membrane dish (35 $\mathrm{mm}$ in diameter, JEOL, Tokyo, Japan). After incubating the cells on the dish for 6-10 h, we removed the planktonic and weakly attached cells by vigorous pipetting in $3 \mathrm{~mL}$ of sterile phosphate-buffered saline (PBS) buffer, and we fixed the remaining adhered cells in $1 \mathrm{~mL}$ of $1 \mathrm{v} / \mathrm{v}$ glutaraldehyde in double distilled water (hereafter DDW) at RT for $10 \mathrm{~min}$. We then quenched the fixing reaction by washing the specimens in $50 \mathrm{mM}$ ammonium chloride followed by an additional washing in DDW. Secreted nanofibrils were visualized by staining with positively charged nanogold (PCG) and phosphotungstic acid (PTA). The DDW-washed specimens were incubated with a $6 \mu \mathrm{M}$ PCG solution (Nanoprobes, Yaphank, NY, USA) for $20 \mathrm{~min}$ at RT; this was repeated once. The specimens were then washed in DDW, and the size of the gold nanoparticles was increased by gold enhancement (GoldEnhance, Nanoprobes) for 10 min at RT, followed by an additional washing in DDW. The resultant specimens were then incubated for $2 \mathrm{~h}$ at RT in $2 \mathrm{v} / \mathrm{v}$ PTA (TAAB Laboratories Equipment, Aldermaston, Berks, England) in DDW. After a final DDW-wash, specimens were soaked in $1 \mathrm{v} / \mathrm{v}$ ascorbic acid in DDW and observed using ASEM at an acceleration voltage of $30 \mathrm{kV}$.

\section{ASSOCIATED CONTENT}

\section{Supporting Information}

This material is available free of charge via the Internet at: . The Supporting Information is available free of charge at https:// pubs.acs.org/doi/10.1021/acsnano.9b04663.

Figure S1: Microscopic images of DNA-stained cells in large aggregates cultured in a batch-culture. Figure S2: A schematic of the quasi-2D microfluidic chamber. Figure S3: Kymograph of an elongating filament. Figure S4: Fluorescent staining of $\mathrm{NH}_{2}$ groups in the nanofibrils. Figure S5: Fluorescent co-staining of sugars and $\mathrm{NH}_{2}$ groups in the nanofibrils. Figure S6: Additional ASEM images of SP-6 and SP-6SL cells. Figure S7: The radius of curvature measured as a function of measured for collisions between filaments and obstacles. Figure S8: Fluorescent staining of cytoplasmic membrane of SP-6 cells (PDF)

Movie S1: A representative time lapse video of SP-6 cells (AVI)

Movie S2: Tracking the centroid of SP-6 cells at the bottom of a glass bottom dish (AVI)

Movie S3: A representative time lapse video of SP-6 SL cells (AVI)

Movie S4: The time lapse video of a bending cell filaments (AVI)

Movie S5: The time lapse video of a reversing filaments (AVI)

\section{AUTHOR INFORMATION}

\section{Corresponding Authors}

*Email: kuno.tatsuki.gb@u.tsukuba.ac.jp.

*Email: nomura.nobuhiko.ge@u.tsukuba.ac.jp.

*Email: utada.andrew.gm@u.tsukuba.ac.jp.

ORCID

Tatsuki Kunoh: 0000-0002-8423-2903

Kana Morinaga: 0000-0001-6739-7514

Shinya Sugimoto: 0000-0002-3114-8513

Andrew S. Utada: 0000-0003-4542-6315

\section{Author Contributions}

${ }^{\perp}$ These authors contributed equally.

Notes

The authors declare no competing financial interest. 


\section{ACKNOWLEDGMENTS}

We thank T. Yamamoto for technical support and J. Fattaccioli, A. M. Spiesser, and D. P. Williford for a critical reading of the manuscript. We acknowledge financial support from Japan Science and Technology Agency (JST), ERATO (JPMJER1502) (N.N.), and the Cooperative Research Project Program of TARA Center, University of Tsukuba (T.K.). A.S.U., N.N., and K.M. are supported by grant-in-aid for Young Scientists (B) (17K15410), Scientific Research (S) (16H06382), and Scientific Research (16J00487) from the Japanese Society for the Promotion of Science (JSPS), respectively.

\section{REFERENCES}

(1) Takeda, M.; Kawasaki, Y.; Umezu, T.; Shimura, S.; Hasegawa, M.; Koizumi, J. Patterns of Sheath Elongation, Cell Proliferation, and Manganese(II) Oxidation in Leptothrix cholodnii. Arch. Microbiol. 2012, 194, 667-673.

(2) Chan, C. S.; McAllister, S. M.; Leavitt, A. H.; Glazer, B. T.; Krepski, S. T.; Emerson, D. The Architecture of Iron Microbial Mats Reflects the Adaptation of Chemolithotrophic Iron Oxidation in Freshwater and Marine Environments. Front. Microbiol. 2016, 7, 796.

(3) Hornlein, C.; Confurius-Guns, V.; Stal, L. J.; Bolhuis, H. Daily Rhythmicity in Coastal Microbial Mats. npj Biofilms Microbiomes 2018, 4,11 .

(4) Spring, S., The Genera Leptothrix and Sphaerotilus. In Prokaryotes: A Handbook on the Biology of Bacteria, 3rd ed.; Rosenberg, E., DeLong, E. F., Lory, S., Stackebrandt, E., Thompson, F., Eds.; Springer-Verlag: Berlin Heidelberg, 2006; Vol. 5, pp 758-777.

(5) Emerson, D.; Fleming, E. J.; McBeth, J. M. Iron-Oxidizing Bacteria: An Environmental and Genomic Perspective. Annu. Rev. Microbiol. 2010, 64, 561-583.

(6) Krumbein, W. E.; Brehm, U.; Gerdes, G.; Gorbushina, A. A.; Levit, G.; Palinska, K. A., Biofilm, Biodictyon, Biomat Microbialites, Oolites, Stromatolites Geophysiology, Global Mechanism, Parahistology. In Fossil and Recent Biofilms; Krumbein, W. E., Paterson, D. M., Zavarzin, G. A., Eds.; Springer: Dordrecht, 2003; pp 1-27.

(7) Emerson, D.; Ghiorse, W. C. Isolation, Cultural Maintenance, and Taxonomy of a Sheath-Forming Strain of Leptothrix discophora and Characterization of Manganese-Oxidizing Activity Associated with the Sheath. Appl. Environ. Microbiol. 1992, 58, 4001-4010.

(8) Vesenka, J.; Havu, J.; Hruby, K.; Emerson, D. A Model for Sheath Formation Coupled to Motility in Leptothrix ochracea. Geomicrobiol. J. 2018, 35, 366-374.

(9) Corstjens, P. L.; de Vrind, J. P.; Westbroek, P.; de Vrind-de Jong, E. W. Enzymatic Iron Oxidation by Leptothrix discophora: Identification of an Iron-Oxidizing Protein. Appl. Environ. Microbiol. 1992, 58, 450454.

(10) Van Veen, W.; Mulder, E.; Deinema, M. H. The SphaerotilusLeptothrix Group of Bacteria. Microbiol. Rev. 1978, 42, 329-356.

(11) Hashimoto, H.; Kobayashi, G.; Sakuma, R.; Fujii, T.; Hayashi, N.; Suzuki, T.; Kanno, R.; Takano, M.; Takada, J. Bacterial Nanometric Amorphous Fe-Based Oxide: A Potential Lithium-Ion Battery Anode Material. ACS Appl. Mater. Interfaces 2014, 6, 5374-5378.

(12) Lovley, D. R. Happy Together: Microbial Communities that Hook Up to Swap Electrons. ISME J. 2017, 11, 327-336.

(13) Nielsen, L. P.; Risgaard-Petersen, N.; Fossing, H.; Christensen, P. B.; Sayama, M. Electric Currents Couple Spatially Separated Biogeochemical Processes in Marine Sediment. Nature 2010, 463, 1071-1074.

(14) Pfeffer, C.; Larsen, S.; Song, J.; Dong, M.; Besenbacher, F.; Meyer, R. L.; Kjeldsen, K. U.; Schreiber, L.; Gorby, Y. A.; El-Naggar, M. Y.; Leung, K. M.; Schramm, A.; Risgaard-Petersen, N.; Nielsen, L. P. Filamentous Bacteria Transport Electrons Over Centimetre Distances. Nature 2012, 491, 218-221.
(15) Chan, C. S.; Fakra, S. C.; Edwards, D. C.; Emerson, D.; Banfield, J. F. Iron Oxyhydroxide Mineralization on Microbial Extracellular Polysaccharides. Geochim. Cosmochim. Acta 2009, 73, 3807-3818.

(16) Kunoh, T.; Hashimoto, H.; McFarlane, I. R.; Hayashi, N.; Suzuki, T.; Taketa, E.; Tamura, K.; Takano, M.; El-Naggar, M. Y.; Kunoh, H.; Takada, J. Abiotic Deposition of Fe Complexes onto Leptothrix Sheaths. Biology 2016, 5, 26.

(17) Kunoh, T.; Hashimoto, H.; Suzuki, T.; Hayashi, N.; Tamura, K.; Takano, M.; Kunoh, H.; Takada, J. Direct Adherence of Fe (III) Particles Onto Sheaths of Leptothrix sp. Strain OUMS1 in Culture. Minerals 2016, 6, 4.

(18) Kunoh, T.; Matsumoto, S.; Nagaoka, N.; Kanashima, S.; Hino, K.; Uchida, T.; Tamura, K.; Kunoh, H.; Takada, J. Amino Group in Leptothrix Sheath Skeleton Is Responsible for Direct Deposition of $\mathrm{Fe}$ (III) Minerals onto the Sheaths. Sci. Rep. 2017, 7, 6498.

(19) Takeda, M.; Kondo, K.; Yamada, M.; Koizumi, J.; Mashima, T.; Matsugami, A.; Katahira, M. Solubilization and Structural Determination of a Glycoconjugate Which Is Assembled into the Sheath of Leptothrix cholodnii. Int. J. Biol. Macromol. 2010, 46, 206-211.

(20) Kunoh, T.; Nakanishi, M.; Kusano, Y.; Itadani, A.; Ando, K.; Matsumoto, S.; Tamura, K.; Kunoh, H.; Takada, J. Biosorption of Metal Elements by Exopolymer Nanofibrils Excreted From Leptothrix Cells. Water Res. 2017, 122, 139-147.

(21) Kunoh, T.; Kusano, Y.; Takeda, M.; Nakanishi, M.; Matsumoto, S.; Suzuki, I.; Takano, M.; Kunoh, H.; Takada, J. Formation of Gold Particles via Thiol Groups on Glycoconjugates Comprising the Sheath Skeleton of Leptothrix. Geomicrobiol. J. 2019, 36, 251-260.

(22) Ema, T.; Miyazaki, Y.; Taniguchi, T.; Takada, J. Robust Porphyrin Catalysts Immobilized on Biogenous Iron Oxide for the Repetitive Conversions of Epoxides and $\mathrm{CO} 2$ Into Cyclic Carbonates. Green Chem. 2013, 15, 2485-2492.

(23) Hashimoto, H.; Asaoka, H.; Nakano, T.; Kusano, Y.; Ishihara, H.; Ikeda, Y.; Nakanishi, M.; Fujii, T.; Yokoyama, T.; Horiishi, N.; Nanba, T.; Takada, J. Preparation, Microstructure, and Color Tone of Microtubule Material Composed of Hematite/Amorphous-Silicate Nanocomposite from Iron Oxide of Bacterial Origin. Dyes Pigm. 2012, 95, 639-643.

(24) Kunoh, T.; Kunoh, H.; Takada, J. Perspectives on the Biogenesis of Iron Oxide Complexes Produced by Leptothrix, an Iron-Oxidizing Bacterium and Promising Industrial Applications for Their Functions. J. Microb. Biochem. Technol. 2015, 7, 419-426.

(25) Fleming, E. J.; Langdon, A. E.; Martinez-Garcia, M.; Stepanauskas, R.; Poulton, N. J.; Masland, E. D. P.; Emerson, D. What's New Is Old: Resolving the Identity of Leptothrix ochracea Using Single Cell Genomics, Pyrosequencing and FISH. PLoS One 2011, 6, No. e17769.

(26) Fleming, E.; Woyke, T.; Donatello, R.; Kuypers, M.; Sczyrba, A.; Littmann, S.; Emerson, D. Insights Into the Fundamental Physiology of the Uncultured Fe-Oxidizing Bacterium Leptothrix ochracea. Appl. Environ. Microbiol. 2018, 84, No. e02239.

(27) Furutani, M.; Suzuki, T.; Ishihara, H.; Hashimoto, H.; Kunoh, H.; Takada, J. Initial Assemblage of Bacterial Saccharic Fibrils and Element Deposition to Form an Immature Sheath in Cultured Leptothrix sp Strain OUMS1. Minerals 2011, 1, 157-166.

(28) Emerson, D.; Ghiorse, W. C. Role of Disulfide Bonds in Maintaining the Structural Integrity of the Sheath of Leptothrix discophora SP-6. J. Bacteriol. 1993, 175, 7819-7827.

(29) Emerson, D.; Ghiorse, W. C. Ultrastructure and ChemicalComposition of the Sheath of Leptothrix discophora SP-6. J. Bacteriol. 1993, 175, 7808-7818.

(30) Ishihara, H.; Suzuki, T.; Hashimoto, H.; Kunoh, H.; Takada, J. Initial Parallel Arrangement of Extracellular Fibrils Holds a Key for Sheath Frame Construction by Leptothrix sp. Strain OUMS1. Minerals 2013, 3, 73-81.

(31) Hol, F. J. H.; Dekker, C. Zooming in to See the Bigger Picture: Microfluidic and Nanofabrication Tools to Study Bacteria. Science 2014, 346, 1251821. 
(32) Nagy, K.; Abraham, A.; Keymer, J. E.; Galajda, P. Application of Microfluidics in Experimental Ecology: The Importance of Being Spatial. Front. Microbiol. 2018, 9, 496.

(33) Aleklett, K.; Kiers, E. T.; Ohlsson, P.; Shimizu, T. S.; Caldas, V. E.; Hammer, E. C. Build Your Own Soil: Exploring Microfluidics to Create Microbial Habitat Structures. ISME J. 2018, 12, 312-319.

(34) Volfson, D.; Cookson, S.; Hasty, J.; Tsimring, L. S. Biomechanical Ordering of Dense Cell Populations. Proc. Natl. Acad. Sci. U. S. A. 2008, 105, 15346-15351.

(35) Grunberger, A.; Paczia, N.; Probst, C.; Schendzielorz, G.; Eggeling, L.; Noack, S.; Wiechert, W.; Kohlheyer, D. A Disposable Picolitre Bioreactor for Cultivation and Investigation of Industrially Relevant Bacteria on the Single Cell Level. Lab Chip 2012, 12, 20602068.

(36) Cho, H.; Jönsson, H.; Campbell, K.; Melke, P.; Williams, J. W.; Jedynak, B.; Stevens, A. M.; Groisman, A.; Levchenko, A. SelfOrganization in High-Density Bacterial Colonies: Efficient Crowd Control. PLoS Biol. 2007, 5, No. e302.

(37) Kunoh, T.; Nagaoka, N.; McFarlane, I. R.; Tamura, K.; ElNaggar, M. Y.; Kunoh, H.; Takada, J. Dissociation and Re-Aggregation of Multicell-Ensheathed Fragments Responsible for Rapid Production of Massive Clumps of Leptothrix Sheaths. Biology 2016, 5, 32.

(38) Bennett, R. R.; Lee, C. K.; De Anda, J.; Nealson, K. H.; Yildiz, F. H.; O’Toole, G. A.; Wong, G. C.; Golestanian, R. Species-Dependent Hydrodynamics of Flagellum-Tethered Bacteria in Early Biofilm Development. J. R. Soc., Interface 2016, 13, 20150966.

(39) De Anda, J.; Lee, E. Y.; Lee, C. K.; Bennett, R. R.; Ji, X.; Soltani, S.; Harrison, M. C.; Baker, A. E.; Luo, Y.; Chou, T.; et al. High-Speed "4D" Computational Microscopy of Bacterial Surface Motility. ACS Nano 2017, 11, 9340-9351.

(40) Adams, L. F.; Ghiorse, W. C. Influence of Manganese on Growth of a Sheathless Strain of Leptothrix discophora. Appl. Environ. Microbiol. 1985, 49, 556-562.

(41) Sauer, K.; Camper, A. K.; Ehrlich, G. D.; Costerton, J. W.; Davies, D. G. Pseudomonas aeruginosa Displays Multiple Phenotypes During Development As a Biofilm. J. Bacteriol. 2002, 184, 1140-1154.

(42) Hinsa, S. M.; Espinosa-Urgel, M.; Ramos, J. L.; O’Toole, G. A. Transition from Reversible to Irreversible Attachment During Biofilm Formation by Pseudomonas fluorescens WCS365 Requires an ABC Transporter and a Large Secreted Protein. Mol. Microbiol. 2003, 49, 905-918.

(43) Tuson, H. H.; Weibel, D. B. Bacteria-Surface Interactions. Soft Matter 2013, 9, 4368-4380.

(44) Brangwynne, C. P.; MacKintosh, F. C.; Kumar, S.; Geisse, N. A.; Talbot, J.; Mahadevan, L.; Parker, K. K.; Ingber, D. E.; Weitz, D. A. Microtubules Can Bear Enhanced Compressive Loads in Living Cells Because of Lateral Reinforcement. J. Cell Biol. 2006, 173, 733-741.

(45) Duvernoy, M.-C.; Mora, T.; Ardré, M.; Croquette, V.; Bensimon, D.; Quilliet, C.; Ghigo, J.-M.; Balland, M.; Beloin, C.; Lecuyer, S.; Desprat, N. Asymmetric Adhesion of Rod-Shaped Bacteria Controls Microcolony Morphogenesis. Nat. Commun. 2018, 9, 1120.

(46) Landau, L. D.; Lifshitz, E. M. Theory of Elasticity, 3rd ed.; Elsevier: New York, 1986; Vol. 7.

(47) Amir, A.; Babaeipour, F.; McIntosh, D. B.; Nelson, D. R.; Jun, S. Bending Forces Plastically Deform Growing Bacterial Cell Walls. Proc. Natl. Acad. Sci. U. S. A. 2014, 111, 5778-5783.

(48) Wang, S.; Arellano-Santoyo, H.; Combs, P. A.; Shaevitz, J. W. Actin-Like Cytoskeleton Filaments Contribute to Cell Mechanics in Bacteria. Proc. Natl. Acad. Sci. U. S. A. 2010, 107, 9182-9185.

(49) Kawasaki, Y.; Kurosaki, K.; Kan, D.; Borges, I. K.; Atagui, A. S.; Sato, M.; Kondo, K.; Katahira, M.; Suzuki, I.; Takeda, M. Identification and Characterization of the S-Layer Formed on the Sheath of Thiothrix nivea. Arch. Microbiol. 2018, 200, 1257-1265.

(50) Suzuki, T.; Kanagawa, T.; Kamagata, Y. Identification of a Gene Essential for Sheathed Structure Formation in Sphaerotilus natans, a Filamentous Sheathed Bacterium. Appl. Environ. Microbiol. 2002, 68, 365-371.

(51) Kawasaki, Y.; Endo, T.; Fujiwara, A.; Kondo, K.; Katahira, M.; Nittami, T.; Sato, M.; Takeda, M. Elongation Pattern and Fine
Structure of the Sheaths Formed by Thiothrix nivea and Thiothrix fructosivorans. Int. J. Biol. Macromol. 2017, 95, 1280-1288.

(52) Kondo, K.; Umezu, T.; Shimura, S.; Narizuka, R.; Koizumi, J.-i.; Mashima, T.; Katahira, M.; Takeda, M. Structure of PerosamineContaining Polysaccharide, a Component of the Sheath of Thiothrix fructosivorans. Int. J. Biol. Macromol. 2013, 59, 59-66.

(53) Stanley, C. E.; Grossmann, G.; Casadevall i Solvas, X.; deMello, A. J. Soil-On-A-Chip: Microfluidic Platforms for Environmental Organismal Studies. Lab Chip 2016, 16, 228-241.

(54) Nelson, Y. M.; Lion, L. W.; Shuler, M. L.; Ghiorse, W. C. Effect of Oxide Formation Mechanisms on Lead Adsorption by Biogenic Manganese (Hydr)Oxides, Iron (Hydr)Oxides, and Their Mixtures. Environ. Sci. Technol. 2002, 36, 421-425.

(55) Katsoyiannis, I. A.; Zouboulis, A. I. Application of Biological Processes for the Removal of Arsenic From Groundwaters. Water Res. 2004, 38, 17-26.

(56) Ghiorse, W. Biology of Iron- and Manganese-Depositing Bacteria. Annu. Rev. Microbiol. 1984, 38, 515-550.

(57) Rhoads, A.; Beyenal, H.; Lewandowski, Z. Microbial Fuel Cell Using Anaerobic Respiration as an Anodic Reaction and Biomineralized Manganese as a Cathodic Reactant. Environ. Sci. Technol. 2005, 39, $4666-4671$.

(58) Yan, X.; Zhou, Q.; Vincent, M.; Deng, Y.; Yu, J.; Xu, J.; Xu, T.; Tang, T.; Bian, L.; Wang, Y.-X. J.; et al. Multifunctional Biohybrid Magnetite Microrobots for Imaging-Guided Therapy. Science Robotics 2017, 2, eaaq1155.

(59) Bente, K.; Codutti, A.; Bachmann, F.; Faivre, D. Biohybrid and Bioinspired Magnetic Microswimmers. Small 2018, 14, 1704374.

(60) Sanchez, S.; Solovev, A. A.; Schulze, S.; Schmidt, O. G. Controlled Manipulation of Multiple Cells Using Catalytic Microbots. Chem. Commun. 2011, 47, 698-700.

(61) Son, S. J.; Reichel, J.; He, B.; Schuchman, M.; Lee, S. B. Magnetic Nanotubes for Magnetic-Field-Assisted Bioseparation, Biointeraction, and Drug Delivery. J. Am. Chem. Soc. 2005, 127, 7316-7317.

(62) Qin, D.; Xia, Y.; Whitesides, G. M. Soft Lithography for Microand Nanoscale Patterning. Nat. Protoc. 2010, 5, 491-502.

(63) Rotem, A.; Abate, A. R.; Utada, A. S.; Van Steijn, V.; Weitz, D. A. Drop Formation in Non-Planar Microfluidic Devices. Lab Chip 2012, $12,4263-4268$.

(64) Strathmann, M.; Wingender, J.; Flemming, H.-C. Application of Fluorescently Labelled Lectins for the Visualization and Biochemical Characterization of Polysaccharides in Biofilms of Pseudomonas aeruginosa. J. Microbiol. Methods 2002, 50, 237-248.

(65) Toda, N.; Doi, A.; Jimbo, A.; Matsumoto, I.; Seno, N. Interaction of Sulfated Glycosaminoglycans with Lectins. J. Biol. Chem. 1981, 256, $5345-5349$

(66) Takeda, M.; Makita, H.; Ohno, K.; Nakahara, Y.; Koizumi, J.-i. Structural Analysis of the Sheath of a Sheathed Bacterium, Leptothrix cholodnii. Int. J. Biol. Macromol. 2005, 37, 92-98.

(67) Sugimoto, S.; Okuda, K.; Miyakawa, R.; Sato, M.; Arita-Morioka, K.; Chiba, A.; Yamanaka, K.; Ogura, T.; Mizunoe, Y.; Sato, C. Imaging of Bacterial Multicellular Behaviour in Biofilms in Liquid by Atmospheric Scanning Electron Microscopy. Sci. Rep. 2016, 6, 25889. 